\title{
Letter to the editor. Spotlight on measles 2010: Timely administration of the first dose of measles vaccine in the context of an ongoing measles outbreak in France
}

A Gagneur (arnaud.gagneur@usherbrooke.ca) ${ }^{1}$, D Pinquier ${ }^{2}$

1. Université de Sherbrooke, CHUS Fleurimont, Département de Pédiatrie. Sherbrooke, Québec, Canada

2. Hôpital Charles Nicolle, Pôle Mère-Femme-Enfant, Centre Hospitalier Universitaire Rouen, France

To the editor: Parent du Châtelet et al. recently described the ongoing measles outbreak in France [1]. We would like to highlight a specific aspect of this outbreak: the significant change in the age distribution of measles cases. In fact, the proportion of cases aged under one year has increased significantly from 2008 to 2010 and this population represents to date the highest incidence rate [1]. Several factors could explain this phenomenon, leading to the question of the necessity of specific control measures in response to the increase of measles cases in the under one year-olds.

During the first year of life, protection against measles is conferred by transferred maternal antibodies. Since the introduction of the measles vaccine, changes in epidemiology have had major effects on the transmission of protective antibodies. The majority of women of childbearing age are now vaccinated and transfer fewer antibodies than naturally immune mothers, conferring protection over a shorter period of time than before to their offspring [2]. A recent French study confirms this fact, showing first that measles antibodies titres were significantly lower in women born after the implementation of the vaccine [2] and secondly that at six months of age, $90 \%$ of infants were not protected whatever the mothers' immunisation status (vaccinated or naturally immune) [3]. Several studies confirm this fact, notably Leuridan et al. demonstrating a median presence of maternal measles antibodies of 3.78 months for infants of naturally immune mothers and 0.97 for infants of vaccinated mothers [4]. Furthermore, the decrease in antibody levels in women of childbearing age may be amplified by three phenomena: first, childbearing age is increasing, with an increased interval between childhood vaccination in the mother and childbirth, resulting in a diminution in antibody levels; and second, boosting by wild type viruses occurs less often as vaccination coverage increases, and this may contribute further to lowering antibody levels in both vaccinated and naturally immune women. In addition, an increasing number of unprotected mothers is being observed, due to failure in catch-up strategies [3].
The result of this early loss of maternal antibodies is the apparition of a critical window of risk for measles infection during the first year of life, which should give rise to several modifications of the measles vaccination programme. One of the barriers to earlier vaccination is the presumed immaturity of the neonatal immunological system. However several studies demonstrate both humoral and cellular responses at an early age [4]. For example, Gans et al. demonstrated priming of infant T-cells with measles antigen as early as six months of age, despite the presence of maternal antibodies [5].

In France, recommendations have been made for vaccination at 12 months of age, and a second dose during the second year of life. Specific recommendations have been made for vaccination at nine months of age for infants in day care centres, with a second dose between 12 to 15 months. In case of contact between infants aged six to eight months and people with measles, vaccination with monovalent vaccine is recommended within 72 hours after contact [3]. Considering that the highest age-specific incidence rate is found in children under one year [1], demonstrating early loss of maternal antibodies, policy makers could consider advancing the measles vaccination programme to, for example, nine months for all infants. In fact, these infants need direct protection until the catch-up vaccination programme can reduce the susceptible population as well as disease transmission.

Early loss of maternal measles antibodies is well documented to date [2-4]. The high number of measles cases in the population under one year of age illustrates this fact. This underscores the importance of timely administration of the first dose of measles vaccine in the context of the ongoing measles outbreak in France and Europe.

\section{References}

1. Parent du Châtelet I, Antona D, Freymuth F, Muscat M, Halftermeyer-Zhou F, Maine C et al. Spotlight on measles 2010: Update on the ongoing measles outbreak in France, 2008-2010. 
Euro Surveill. 2010;15(36):pii=19656. Available from: http:// www.eurosurveillance.org/ViewArticle.aspx?Articleld $=19656$

2. Pinquier D, Gagneur A, Aubert M, Brissaud O, Le Guen CG,

Hau-Rainsard et al. Distribution of serum measles-neutralizing antibodies according to age in women of childbearing age in France in 2005-2006: impact of routine immunization. Pediatr Infect Dis J. 2007;26(8):749-50.

3. Gagneur A, Pinquier D, Aubert M, Soubeyrand B, Balu L, Brissaud $O$ et al. Kinetics of decline of maternal measles virus neutralizing antibodies in sera of infants in France in 2006. Clin Vaccine Immunol. 2008;15(12):1845-50.

4. Leuridan E, Hens N, Hutse V, leven M, Aerts M, Van Damme P. Early waning of maternal measles antibodies in era of measles elimination: longitudinal study. BMJ. 2010;18;340:C1626.

5. Gans HA, Yasukawa LL, Alderson A, Rinki M, DeHovitz R, Beeler $J$ et al. Humoral and cell-mediated immune responses to an early 2 -dose measles vaccination regimen in the United States. J Infect Dis. 2004;190(1):83-90. 